\title{
Fingertip reconstruction with a subcutaneous flap and composite graft composed of nail bed and volar pulp skin
}

\author{
Sung Hoon Koh ${ }^{1}$, Ilou Park ${ }^{1}$, Jin Soo Kim ${ }^{1}$, Dong Chul Lee ${ }^{1}$, Si Young Roh ${ }^{1}$, Kyung Jin Lee ${ }^{1}$, \\ Min Ki Hong ${ }^{2}$ \\ ${ }^{1}$ Department of Plastic and Reconstructive Surgery, Gwangmyeong Sungae General Hospital, Gwangmyeong; ${ }^{2}$ Department of Plastic and \\ Reconstructive Surgery, International St. Mary's Hospital, Incheon, Korea
}

Background Fingertip injuries are very common; however, the reconstruction of volar pulp defects with nail bed defects is challenging in the absence of the amputated segment. We reconstructed fingertip amputations with nail bed defects using a new surgical approach: a subcutaneous flap and composite graft.

Methods We treated 10 fingertip amputation patients without an amputated segment, with exposed distal phalangeal bone and full-thickness nail bed defects between February 2018 and December 2020. All patients underwent two-stage surgery: in the first stage, a subcutaneous flap was performed to cover the exposed distal phalanx, and in the second stage, a composite graft, consisting of nail bed, hyponychium, and volar pulp skin, was applied over the subcutaneous flap.

Results All flaps survived and all composite grafts were successful. The wounds healed without any significant complications, including the donor site. The average follow-up duration was 11.2 months (range, 3-27 months). The new nail and the shape of the volar pulp were evaluated during follow-up. All patients were satisfied with their natural fingertip shapes and the new nails did not have any serious deformities.

Conclusions A subcutaneous flap in combination with a composite graft fitting the shape of the defect could be another option for fingertip injuries without amputated segments.

Keywords Finger injuries / Nails / Surgical flap
Correspondence: Jin Soo Kim Department of Plastic and Reconstructive Surgery, Gwangmyeong Sungae General Hospital, 36 Digital-ro, Gwangmyeong 14241 , Korea

Tel: +82-2-2680-7637

Fax: +82-2-2615-7218

Email: hlaze@daum.net

Received: July 1, $2021 \bullet$ Revised: August 12, $2021 \bullet$ Accepted: September 30, 2021

pISSN: 2234-6163 • elSSN: 2234-6171 • https://doi.org/10.5999/aps.2021.01200 • Arch Plast Surg 2022;49:70-75

\section{INTRODUCTION}

Fingertip injuries are one of the most frequent types of hand injuries, regardless of age [1,2]. However, reconstruction of a volar pulp defect with loss of the nail bed is challenging. Replantation may be a preferable option if the amputated segment is present. Other reconstructive methods for fingertip amputation, such as the Moberg flap, volar V-Y advancement flap, thenar flap, and small free flap have been verified as good treatments; however, these options cannot reconstruct the nail bed. Therefore, we present a subcutaneous flap and subsequent composite graft composed of nail bed and volar pulp skin, which could be considered in the reconstruction of defects of both the pulp and nail bed. 


\section{METHODS}

This reconstructive method was performed in 10 patients with an exposed distal phalanx and full-thickness nail bed defect between February 2018 and December 2020. All patients were treated with a two-stage surgical procedure, first involving coverage of the exposed distal phalanx using a subcutaneous flap, followed 3 weeks later by a composite graft consisting of nail bed and volar pulp skin from the great toe. We performed this method for cases of fingertip amputation without the amputated segment and patients with severe crush injuries of the fingertip. We also included failed cases of replantation.

\section{Operative technique}

\section{Stage 1: subcutaneous flap}

A subcutaneous flap is used to cover the exposed bone (Fig. 1A). The ulnar and radial digital arteries meet at the lunular level and become the distal transverse palmar arch (DTPA) [3]. More than two branches arise longitudinally from the DTPA, which supply arterial blood up to the fingertip [4]. It is possible to maintain arterial circulation on both sides of the fingertip, even if one of the digital arteries is cut.

If there is a fracture, the distal phalanx is fixed with a Kirschner wire. The nail plate is removed, and the crushed nail bed and pulp tissue are adequately debrided. The size and location of the tissue defect are measured, and the surgeon evaluates which side the skin incision should start from and how large of a flap is necessary.

The longitudinal skin incision starts on the opposite side of the defect around the distal interphalangeal (DIP) joint level. The subdermal dissection is at least half the defect size to elevate a sufficiently large subcutaneous flap. After subdermal dissection, the subcutaneous vein is observed just below the skin flap. It is beneficial to save the subcutaneous veins, but if doing so is not possible, the circulation of the subcutaneous flap will be adequate because the elevated subcutaneous flap also has venae comitantes.
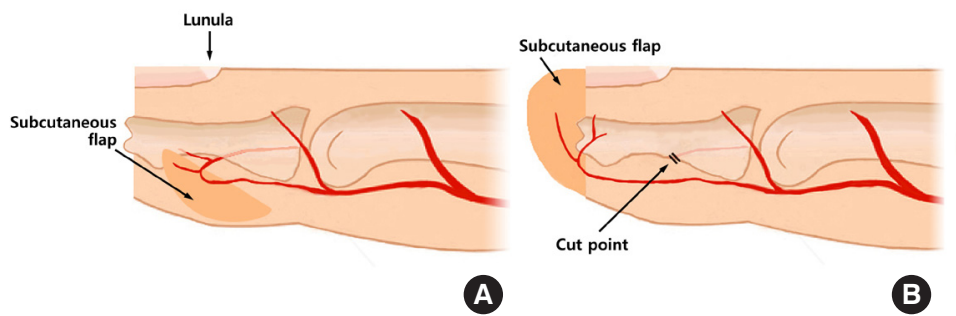

B
More extensive subcutaneous dissection is needed to find a digital artery and nerve in the deeper level of the subcutaneous tissue. If an artery and nerve are detected, sufficient dissection along the subcutaneous plane, only cutting branches of the digital artery, will enable coverage of a small fingertip defect. If the fingertip defect is large, the digital artery should be cut around the DIP joint level, and the subcutaneous flap should include more proximal subcutaneous tissue from the cutting side.

If the defect is small, the digital nerve can be pulled without severing it. If the defect is large, the subcutaneous flap can be transposed with intraneural dissection at the bifurcation of the digital nerve. Nevertheless, if the subcutaneous flap cannot be transferred, the digital nerve should be cut.

After trimming the pedicle, the flap is transposed to cover the exposed bone and nail bed defects [5]. Appropriate rotation toward the defect is necessary, depending on the location and size of the defect. Approximation of the ruptured nail bed is performed, and the flap is sutured in a tension-free manner (Fig. 1B). The flap circulation can be checked by observing the bleeding through the subcutaneous fat tissue. Moist dressing with ointment and a short arm splint are applied after absorbable hemostat is put on the flap.

\section{Stage 2: composite graft}

The composite graft consisting of the nail bed and volar skin is performed roughly 3 weeks after flap surgery. The graft is harvested from a big toe under a digital nerve block and a digital tourniquet. According to the "like-with-like" principle, the composite graft is designed to resemble the defect in terms of location and size (Fig. 1C). Splint-thickness sterile matrix, hyponychium, and volar pulp skin are harvested with continuity and fitted for the shape and size of the defect using the freehand technique. While the nail bed is harvested as deep as the defect of the recipient, the full-thickness volar pulp skin is harvested with sufficient thickness to contain a small amount of subcutaneous tissue. The toenail is reinserted into the eponychial fold of the donor toe, and trephination over the toenail is performed to

Fig. 1. Schematic diagrams of the surgical technique: a subcutaneous flap and composite graft. (A) Tuft bone is exposed with nail bed and volar pulp defect. The subcutaneous flap is elevated, including the distal transverse palmar arch. (B) The amputated fingertip is covered with the subcutaneous flap after cutting a digital artery. (C) The composite tissue is harvested from the great toe, and grafted over the subcutaneous flap of the finger. 
prevent subungual hematoma. The composite tissue is grafted and sutured on the recipient fingertip defect previously treated with a subcutaneous flap (Fig. 1C), followed by a tie-over dressing. The tie-over dressing is removed 7 days postoperatively.

\section{RESULTS}

The average age of the 10 patients was 42.4 years (range, 2-61 years) with eight men and two women. All injured fingers were crushed and had Allen classification type 3 amputations with significant exposure of the distal phalanx, as well as defects in both the nail bed and pulp area [6]. Of the 10 fingers treated, there were two thumbs, four index fingers, and four middle fin- gers. Eight patients received the subcutaneous flap as an initial operation due to the absence of the amputated segment, and two patients underwent this flap surgery as a secondary operation due to necrosis after replantation (Table 1). Fractures of the distal phalanx were present in two cases.

All flaps survived and all composite grafts were successful. Two cases of tuft fractures healed well. We examined the shape of the pulp area and nail growth, and the average follow-up was 11.2 months (range, 4-27 months). The recipient and donor sites healed without any significant complications. Although the donor site usually had a subungual hematoma in the immediate postoperative period, none had significant morbidity during long-term follow-up. The nails of all patients did not have any

Table 1. Patient data

\begin{tabular}{|c|c|c|c|c|c|c|c|c|c|}
\hline $\begin{array}{c}\text { Patient } \\
\text { no. }\end{array}$ & Sex/age (yr) & $\begin{array}{l}\text { Injured } \\
\text { finger }\end{array}$ & $\begin{array}{l}\text { Reconstructive } \\
\text { type }\end{array}$ & Cause of trauma & $\begin{array}{l}\text { Size of flap } \\
(\mathrm{cm})\end{array}$ & $\begin{array}{l}\text { Size of graft } \\
(\mathrm{cm})\end{array}$ & $\begin{array}{l}\text { Interval between } \\
\text { operations (wk) }\end{array}$ & $\begin{array}{c}\text { Donor site } \\
\text { of graft }\end{array}$ & $\begin{array}{l}\text { Complications } \\
\text { at the donor site }\end{array}$ \\
\hline 1 & $M / 57$ & Rt. index & Immediate & Jamming in a door & $1.3 \times 0.8$ & $1.3 \times 0.8$ & 3 & Rt. big toe & None \\
\hline 2 & $\mathrm{~F} / 50$ & Lt. index & Immediate & Press machine & $1.5 \times 0.8$ & $1.5 \times 1.0$ & 3 & Rt. big toe & None \\
\hline 3 & $M / 5$ & Rt. middle & Delayed & Animal bite & $0.8 \times 0.8$ & $0.8 \times 0.8$ & 3 & Rt. big toe & None \\
\hline 4 & $M / 2$ & Lt. index & Immediate & Jamming in a moving walkway & $0.7 \times 0.6$ & $0.7 \times 0.6$ & 3 & Lt. big toe & None \\
\hline 5 & $F / 61$ & Lt. index & Failed replantation & Press machine & $1.2 \times 0.6$ & $1.2 \times 1.0$ & 4 & Lt. big toe & None \\
\hline 6 & $M / 50$ & Rt. middle & Immediate & Press machine & $1.2 \times 1.0$ & $1.2 \times 1.0$ & 3 & Rt. big toe & None \\
\hline 7 & $M / 42$ & Rt. middle & Immediate & Press machine & $1.0 \times 0.8$ & $1.0 \times 0.8$ & 3 & Rt. big toe & None \\
\hline 8 & $M / 57$ & Lt. thumb & Immediate & Press machine & $1.7 \times 0.7$ & $2.0 \times 1.5$ & 3 & Lt. big toe & None \\
\hline 9 & $\mathrm{M} / 46$ & Rt. middle & Failed replantation & Press machine & $1.1 \times 1.0$ & $1.1 \times 1.0$ & 4 & Lt. big toe & None \\
\hline 10 & $M / 54$ & Rt. thumb & Immediate & Press machine & $1.4 \times 1.0$ & $1.2 \times 1.0$ & 6 & Rt. big toe & None \\
\hline
\end{tabular}

M, male; F, female; Rt., right; Lt., left.

Table 2. Evaluation of nail bed reconstruction according to Zook's criteria

\begin{tabular}{|c|c|c|c|c|c|c|c|c|c|c|c|c|}
\hline \multirow{2}{*}{ Variation } & & & \multicolumn{10}{|c|}{ Patient no. } \\
\hline & & & 1 & 2 & 3 & 4 & 5 & 6 & 7 & 8 & 9 & 10 \\
\hline \multirow[t]{5}{*}{ Nail shape } & Identical & & $\bullet$ & & $\bullet$ & $\bullet$ & & $\bullet$ & & $\bullet$ & & - \\
\hline & Shorter & Minor & & $\bullet$ & & & $\bullet$ & & $\bullet$ & & $\bullet$ & \\
\hline & Narrower & Minor & & & & & & & & & & \\
\hline & Longitudinal curve & Minor & & & & & & & & & & \\
\hline & Transverse curve & Minor & & & & & & & & & & \\
\hline \multirow[t]{3}{*}{ Nail adherence } & Complete & & $\bullet$ & $\bullet$ & $\bullet$ & $\bullet$ & $\bullet$ & $\bullet$ & $\bullet$ & $\bullet$ & $\bullet$ & $\bullet$ \\
\hline & $\geq 2 / 3$ & Minor & & & & & & & & & & \\
\hline & $<2 / 3$ & Major & & & & & & & & & & \\
\hline \multirow[t]{3}{*}{ Eponychium } & Identical & & $\bullet$ & $\bullet$ & $\bullet$ & $\bullet$ & & $\bullet$ & $\bullet$ & $\bullet$ & $\bullet$ & $\bullet$ \\
\hline & Notched & Minor & & & & & & & & & & \\
\hline & Synechia & Minor & & & & & $\bullet$ & & & & & \\
\hline \multirow[t]{5}{*}{ Nail surface } & Identical & & $\bullet$ & $\bullet$ & $\bullet$ & $\bullet$ & & $\bullet$ & $\bullet$ & $\bullet$ & $\bullet$ & \\
\hline & Slightly rough & Minor & & & & & $\bullet$ & & & & & $\bullet$ \\
\hline & Very rough & Major & & & & & & & & & & \\
\hline & Longitudinal ribs & Minor & & & & & & & & & & \\
\hline & Transverse grooves & Minor & & & & & & & & & & \\
\hline \multirow[t]{2}{*}{ Split } & Absent & & $\bullet$ & $\bullet$ & $\bullet$ & $\bullet$ & $\bullet$ & $\bullet$ & $\bullet$ & $\bullet$ & $\bullet$ & $\bullet$ \\
\hline & Present & Major & & & & & & & & & & \\
\hline \multirow[t]{2}{*}{ Total } & & Major & 0 & 0 & 0 & 0 & 0 & 0 & 0 & 0 & 0 & 0 \\
\hline & & Minor & 0 & 1 & 0 & 0 & 3 & 0 & 1 & 0 & 1 & 1 \\
\hline Grade & & & A & B & $A$ & A & $D$ & $A$ & B & $A$ & B & B \\
\hline
\end{tabular}

Grade A: excellent (no variations), grade B: very good (1 minor), grade C: good (2 minor), grade D: fair (3 minor or 1 major), grade E: poor ( $>3$ minor or $>1$ major). 
serious deformity such as nail ridge, split nail, pterygium, or hooked nail, except for one patient (patient no. 5) who had loss of almost the entire nail bed and distal phalanx after replantation failure. The outcomes of nail growth were excellent in five patients (50\%), very good in four patients (40\%), and fair in one patient (10\%), in accordance with Zook's criteria (Table 2) [7].

\section{Case 1}

A 50-year-old woman presented with amputation of her left index finger by a press machine. The amputated segment was small and severely crushed, and we decided to operate with a subcutaneous flap for the exposed distal phalanx. She underwent composite graft surgery 3 weeks later. At a 27-month follow-up, a minor variation of the shortened nail was observed, and the nail was graded as "very good" (Fig. 2).

\section{Case 2}

A 5-year-old boy was involved in a horse bite accident, leading to necrosis of the right middle fingertip. After debridement, a soft-tissue defect of the fingertip was observed with an exposed distal phalanx. A subcutaneous flap was applied, and a subsequent composite graft was performed 3 weeks after flap surgery. At a 12-month follow-up, the new nail was graded as "excellent" (Fig. 3A).

\section{DISCUSSION}

Fingertip injuries are among the most common hand injuries
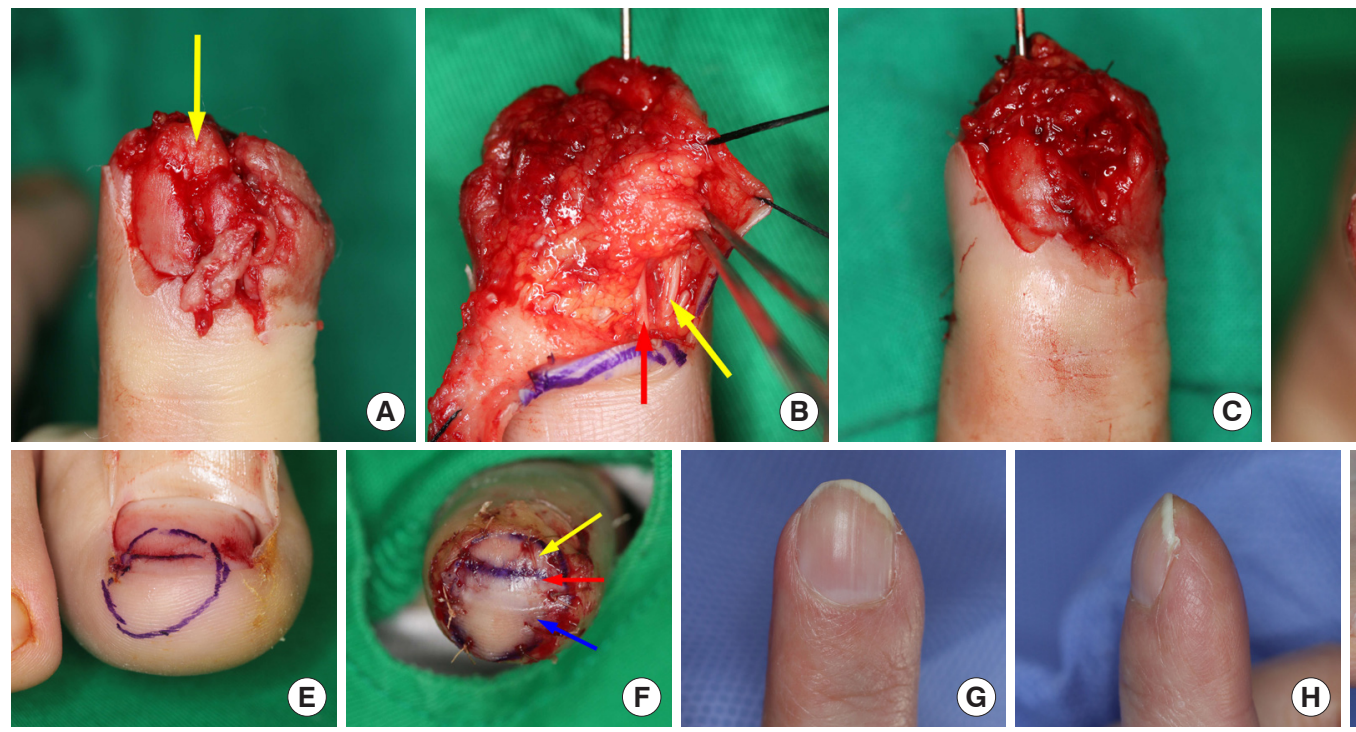

(H)
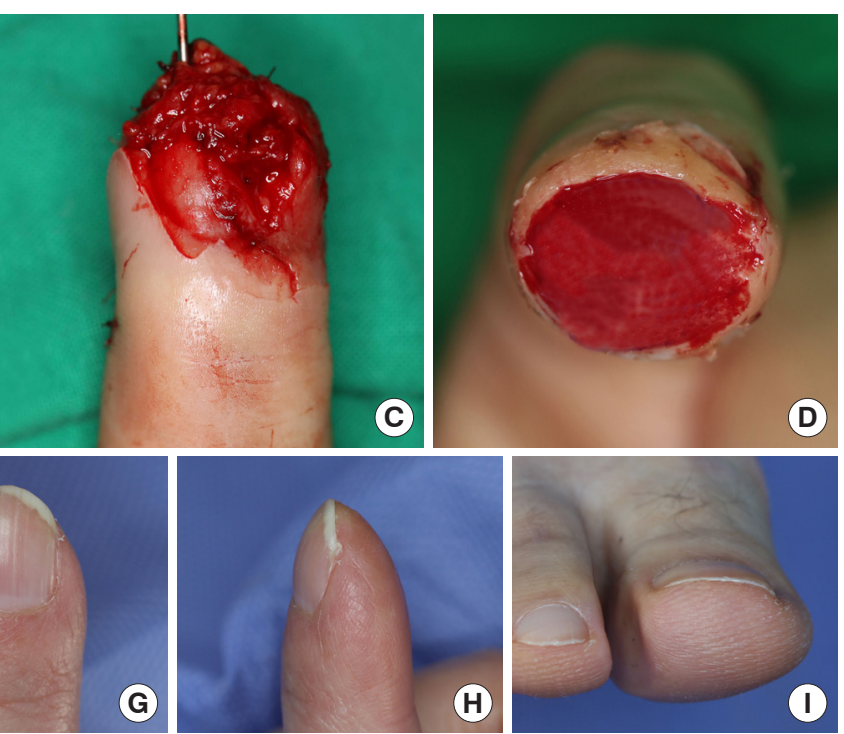

Fig. 2. Preoperative, intraoperative, and long-term follow-up photographs (case 1). (A) The tip of distal phalanx bone is exposed (yellow arrow) with a nail bed defect. (B) A digital artery (red arrow) and nerve (yellow arrow) are exposed around the distal interphalangeal joint level. (C) The subcutaneous flap is fully covered up to the bone exposure of the nail bed. (D) The subcutaneous flap maintains the coverage of the volar and dorsal sides of the fingertip defect 3 weeks after flap surgery. (E) A composite tissue graft is designed on the fibular side of the great toe according to the defect and consisting of nail bed, hyponychium, and pulp skin. (F) The fingertip defect is covered with the three-component tissue: nail bed (yellow arrow), hyponychium (red arrow), and pulp skin (blue arrow). The dorsal $(\mathrm{G})$ and lateral view $(\mathrm{H})$ of the reconstructed finger. A minor scar is seen without any nail deformity 27 months after the fingertip reconstruction. (I) No complication is seen at the donor site of the composite graft at a 27-month follow-up.
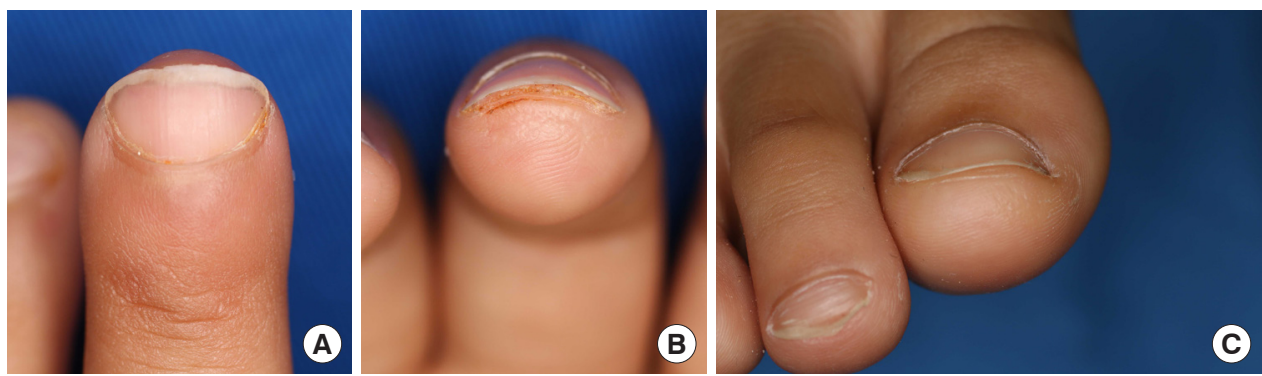

Fig. 3. Long-term follow-up photographs (case 2). The dorsal (A) and tip view (B) of the healed finger. A minor scar is seen without any nail deformity at a 12-month follow-up. (C) No complication is seen at the donor site of the composite graft at a 12-month follow-up. 
regardless of age, as the fingertip is a prominent and frequently used part of the hand $[1,2]$. However, no consensus yet exists regarding the ideal reconstruction of fingertip injuries with nail bed defects, and its reconstructive methods are limited.

Fingertip injuries are managed according to the Allen classification as follows [6]: (1) Type 1 has only a pulp defect without nail bed and bone exposure. If the amputated segment is present, a composite graft is the treatment of choice. If not, secondary healing is usually an adequate option; (2) Type 2 has both nail bed and pulp defects, but without bone exposure. If the amputated segment is present, the treatment depends on the shape of the defect. For the transverse type, acceptable options include various local and regional flaps, such as the thenar flap, Moberg flap, volar V-Y advancement flap, bilateral V-Y advancement flap, and cross-finger flap [8-11]. However, if the shape is oblique, a free flap is recommended; (3) Type 3 has an exposed distal phalanx as well as both nail bed and volar pulp defects. In these cases, replantation or flap surgery is required.

Although conventional fingertip reconstructions such as the volar V-Y advancement flap and the Moberg flap are often applied in cases of transverse amputation over Allen classification type 3 injuries, the Moberg flap is difficult to apply for fingers other than the thumb $[8,9]$. Additionally, although the bilateral $\mathrm{V}-\mathrm{Y}$ advancement flap is suitable for central fingertip defects owing to its limited bilateral advancement, postoperative pain is one of the worst complications related to fingertip scars [10]. These conventional flaps may not be ideal reconstructive methods for nail bed defects.

After the development of acellular dermal matrix (ADM), nail bed reconstruction with $\mathrm{ADM}$ and a split-thickness skin graft (STSG) were recently introduced [11]. Although this method has certain advantages, including minimal functional deficits and donor site morbidity, it may not be suitable for complex crush trauma patients. It also cannot be applied to large defects or volar pulp defects with bone exposure.

Several methods of reconstruction exist for nail bed and volar pulp defects with an exposed distal phalanx. A nail bed graft is possible for the nail bed over the bone. If the nail bed defect is small, a nail bed graft over the bone can be done. However, if the defect is larger, graft survival tends to be low due to the presence of less viable vascularity over the exposed bone $[12,13]$. A nail bed graft cannot be used to reconstruct a volar pulp defect; therefore, it is only an option for the dorsal side of the exposed distal phalanx.

Microvascular toenail transfer involves the transfer of a composite of nail and a finite portion of pulp from the toe. It can be applied from partial to full defects of the fingertip. However, its indications for nail reconstruction are relatively rare, and it re- quires advanced microsurgical techniques [14].

Lee et al. [15] introduced a thin thenar fascial flap for coverage of exposed bone with subsequent nail bed grafts. The fascial flap provides a healthy wound bed for nail bed grafting. However, this method may induce joint stiffness due to immobilization before flap division.

Thus, we propose a subcutaneous flap to cover the exposed distal phalanx and a composite graft composed of nail bed, hyponychium, and volar skin. The composite graft was taken from the fibular hyponychial side of the great toe to decrease irritation around the donor site when patients wore their shoes. The donor site healed by secondary intention in the first four cases. However, the patient complained of pain during dressing of the donor site; therefore, we used an additional STSG from the fibular side of the big toe. The thin STSG was used as the donor for the composite graft in the last six cases. This was done to achieve faster healing of the donor site of the composite graft and to reduce pain.

The present study has limitations in that it included a relatively small number of cases. It is expected that the subcutaneous tissue of the donor site will be reduced after transposing adjacent volar tissue to the defect site. Four patients complained of insufficient volume of the volar pad compared to normal fingertips. However, no patients reported experiencing pain in the reconstructed finger that caused discomfort in daily life. We suggest that this is because the incision of the subcutaneous flap was on the lateral side (i.e., the non-pinching side of the finger), unlike the volar V-Y advancement flap.

The subcutaneous flap from the adjacent tissue covered the exposed bone sufficiently and became a good recipient bed for the nail bed graft. The reconstructed hyponychial fold is natural because the composite graft was harvested from the nail bed, hyponychium, and pulp skin of the big toe as one piece (Table 2). If the graft is done with an STSG only, excluding the nail bed graft, the possibility of hook nail deformity may be high. While the nail bed was harvested at a depth equal to that of the defect, the volar pulp skin was harvested with a sufficient thickness to contain some subcutaneous tissue. This reconstruction does not require long-term immobilization and minimizes major complications, such as shortened nail, hook nail deformity (Figs. 2G, 3A), and a bulky volar pulp. These techniques require a relatively short learning curve as they are not microsurgical procedures.

The subcutaneous flap was obtained from tissue adjacent to the injured tissue without extensive sacrifice. The donor scar was minimal and was not distinguishable from a traumatic scar (Figs. 2H, 3B). The composite graft required a small and thin volume of tissue from the great toe. No donor site morbidity 
and minimal scarring was observed in the great toe (Figs. 2I, 3C).

A subcutaneous flap and composite graft fitting the defect shape could constitute a new reconstructive paradigm for fingertip injuries with nail bed defects and distal phalanx exposure.

\section{NOTES}

\section{Conflict of interest}

Jin Soo Kim and Dong Chul Lee are editorial board members of the journal but were not involved in the peer reviewer selection, evaluation, or decision process of this article. No other potential conflicts of interest relevant to this article were reported.

\section{Ethical approval}

The study was approved by the Institutional Review Board of Gwangmyeong Sungae General Hospital (IRB No. KIRB2020-N-004) and performed in accordance with the principles of the Declaration of Helsinki. Written informed consent was obtained.

\section{Patient consent}

The patients provided written informed consent for the publication and the use of their images.

\section{Author contribution}

Conceptualization: JS Kim, MK Hong. Data curation: I Park. Formal analysis: I Park. Methodology: SH Koh, DC Lee, SY Roh, KJ Lee, MK Hong. Project administration: SH Koh. Visualization: I Park. Writing - original draft: I Park. Writing - review \& editing: I Park, JS Kim, MK Hong. All authors read and approved the final manuscript.

\section{ORCID}

$\begin{array}{ll}\text { Sung Hoon Koh } & \text { https://orcid.org/0000-0002-8094-2561 } \\ \text { Ilou Park } & \text { https://orcid.org/0000-0002-6856-4895 } \\ \text { Jin Soo Kim } & \text { https://orcid.org/0000-0003-3369-2974 } \\ \text { Dong Chul Lee } & \text { https://orcid.org/0000-0003-4211-6901 } \\ \text { Si Young Roh } & \text { https://orcid.org/0000-0002-8625-6124 } \\ \text { Kyung Jin Lee } & \text { https://orcid.org/0000-0002-9448-8291 } \\ \text { Min Ki Hong } & \text { https://orcid.org/0000-0002-8587-7420 }\end{array}$

\section{REFERENCES}

1. Cheung K, Hatchell A, Thoma A. Approach to traumatic hand injuries for primary care physicians. Can Fam Physician 2013;59:614-8.

2. Satku M, Puhaindran ME, Chong AK. Characteristics of fingertip injuries in children in Singapore. Hand Surg 2015; 20:410-4.

3. Strauch B, de Moura W. Arterial system of the fingers. J Hand Surg Am 1990;15:148-54.

4. Nam YS, Jun YJ, Kim IB, et al. Anatomical study of the fingertip artery in Tamai zone I: clinical significance in fingertip replantation. J Reconstr Microsurg 2017;33:45-8.

5. Park J, Kim JS, Koh SH, et al. Fingertip reconstruction with subcutaneous island flap and composite graft: a case report. Arch Hand Microsurg 2020;25:282-6.

6. Spyropoulou GA, Shih HS, Jeng SF. Free pulp transfer for fingertip reconstruction: the algorithm for complicated $\mathrm{Al}-$ len fingertip defect. Plast Reconstr Surg Glob Open 2016;3: e584.

7. Zook EG, Guy RJ, Russell RC. A study of nail bed injuries: causes, treatment, and prognosis. J Hand Surg Am 1984;9: 247-52.

8. Atasoy E, Ioakimidis E, Kasdan ML, et al. Reconstruction of the amputated finger tip with a triangular volar flap: a new surgical procedure. J Bone Joint Surg Am 1970;52:921-6.

9. Moberg E. Aspects of sensation in reconstructive surgery of the upper extremity. J Bone Joint Surg Am 1964;46:817-25.

10. Kutler W. A new method for finger tip amputation. J Am Med Assoc 1947;133:29.

11. Liu TH, Hsieh MC, Chou PR, et al. Reconstruction for defects of total nail bed and germinal matrix loss with acellular dermal matrix coverage and subsequently skin graft. Medicina (Kaunas) 2020;56:17.

12. Brown RE, Zook EG, Russell RC. Fingertip reconstruction with flaps and nail bed grafts. J Hand Surg Am 1999;24:34551.

13. Shepard GH. Treatment of nail bed avulsions with splitthickness nail bed grafts. J Hand Surg Am 1983;8:49-54.

14. Morrison WA. Microvascular nail transfer. Hand Clin 1990; 6:69-77.

15. Lee KJ, Kim YW, Kim JS, et al. Nail bed defect reconstruction using a thenar fascial flap and subsequent nail bed grafting. Arch Plast Surg 2019;46:57-62. 